\title{
Altendorfer, Laura Maria: Influencer in der digitalen Gesundheitskommunikation. Instagramer, YouTuber und Co. zwischen Qualität, Ethik und Professionalisierung.
}

\author{
Baden-Baden: Nomos 2019. 278 Seiten. Preis: $€ 54$
}

\section{Anja Kalch}

Angenommen: 6. März 2021 / Online publiziert: 15. März 2021

(C) Der/die Autor(en) 2021

Bei der Frage, wo sich die Menschen in Deutschland über Gesundheitsfragen informieren, rangiert das Internet nun schon seit einigen Jahren in Umfragen auf Platz zwei, direkt nach Gesprächen mit den eigenen ÄrztInnen. Das dort verfügbare Portfolio an Gesundheitsinformationen ist inhaltlich nahezu grenzenlos und deckt eine große Bandbreite verschiedenster Professionalisierungsgrade seitens der KommunikatorInnen ab. Während einige Online-Gesundheitsangebote eine direkte Entsprechung zu klassischen Offline-Formaten haben und von journalistischen oder medizinischen ExpertInnen bereitgestellt werden (z. B. Broschüren, Zeitschriftenbeiträge, Gesundheitsdokumentationen in Mediatheken), liegen auch zahlreiche Angebote von InfluencerInnen vor. Laura-Maria Altendorfer hat sich in ihrer hier vorliegenden Dissertation vorgenommen, das Rollenselbstbild von InfluencerInnen zu beschreiben und ausgehend davon zu erforschen, welchen Beitrag diese aus Rezipierendenperspektive für die Gesundheitskommunikation leisten. Angesichts der Vielzahl an gesundheitsbezogenen Kanälen und Beiträgen in sozialen Medien und der hohen Reichweite, die einige InfluencerInnen dort erzielen, erscheint diese Fragestellung hoch relevant.

Zur Beantwortung dieser Fragestellung befasst sich der erste Teil des Buches zunächst nur mit dem Rollenverständnis von InfluencerInnen und geht der Frage nach, welche Charakteristika InfluencerInnen in sozialen Medien haben. Dabei werden viele Bezüge aus der Marktforschung sowie dem Marketing integriert, gleichzeitig werden aber auch kommunikationswissenschaftlich relevante Aspekte wie ethische Kriterien und die Relevanz von Verantwortung betrachtet. Empirisch wird dieser erste Teil der Arbeit mit qualitativen Leitfadeninterviews bearbeitet. Obgleich die

Dr. A. Kalch $(\bowtie)$

Institut für Medien, Wissen und Kommunikation, Universität Augsburg,

Universitätsstraße 10, 86159 Augsburg, Deutschland

E-Mail: anja.kalch@phil.uni-augsburg.de 
Arbeit InfluencerInnen in der Gesundheitskommunikation untersucht, besteht die Stichprobe nur zu einem Teil aus InfluencerInnen, die gesundheitsbezogene Inhalte als Hauptthemengebiete angeben. Schade ist, dass dann die Ergebnisse noch nicht einmal einen Einblick in diese Untergruppe erlauben. Eine solche weite Annäherung an das Forschungsfeld ist ungewöhnlich. Auf diese Weise müssen Fragestellungen zu den Spezifika von GesundheitsinfluencerInnen, zu ihrem fachlichen Hintergrund oder zu Besonderheiten ethischer Kriterien auf zukünftige Forschungsarbeiten verschoben werden.

Erst etwa ab der Hälfte des Bandes wird der Fokus auf die Gesundheitskommunikation gelegt. Auch hier wird wiederum ein allgemeiner Einstieg gewählt, der einen ersten Einblick in eine Vielzahl grundlegender Konzepte und Erkenntnisse der Gesundheitskommunikation, wie beispielsweise Gesundheit und Krankheit, KommunikatorInnen in der Gesundheitskommunikation, Inhalte der Gesundheitskommunikation oder auch die Rezeption von Gesundheitsinhalten und Mediennutzung sowie deren Wirkung, verspricht. Ein derartig umfangreicher Überblick ist im Rahmen einer solchen Arbeit auf wenigen Seiten kaum realisierbar, besonders wenn man bedenkt, dass jeder dieser Themenbereiche ganze Lehr- und Handbücher füllt. Demnach überrascht es auch nicht, dass hier wirklich nur allgemeine Einblicke unter Verweis auf vorliegende, einschlägige Handbücher, Lehrbücher und deutschsprachige Sammelbände gegeben werden. An einigen Stellen vermisst man den stärkeren und tieferen Einbezug aktueller, internationaler Forschungsliteratur.

Anknüpfend an diesen Einstieg in die digitale Gesundheitskommunikation erfolgt in Kapitel 5 ein zweiter empirischer Abschnitt, in dem InfluencerInnen in der digitalen Gesundheitskommunikation aus Sicht von RezipientInnen betrachtet werden. Auch hier führt Altendorfer wieder qualitative Interviews durch, diesmal in narrativer Form, und kombiniert diese im Rahmen eines zweistufigen Verfahrens mit einer quantitativen Inhaltsanalyse von gesundheitsbezogenen InfluencerInnen-Posts in sozialen Medien. Die Sammlung der gesundheitsbezogenen Posts erfolgte im Vorfeld der Interviews durch die StudienteilnehmerInnen. Die dabei erzielten Ergebnisse gewähren einen überblicksartigen Einblick in eine Reihe an Themenfeldern, die aus RezipientInnensicht für die Bewertung der Beiträge relevant sind. Dies umfasst etwa die subjektive Definition von Gesundheit, die Art des Gesundheitsbezugs in den Posts, erwartete Wirkungen, aber auch die Wahrnehmung der werblichen Inhalte. Während zu einzelnen dieser Aspekte in bestimmten Themenfeldern (z.B. der Wahrnehmung von Schönheitsidealen bei Fitnessposts) bereits empirische Studien vorliegen, ist eine solche Gesamtschau, wie sie hier angeboten wird, neu und liefert einen guten Überblick über das Themenfeld.

Spannend ist der Versuch, sowohl die kommunikatorInnenseitige als auch die rezipientInnenseitige Betrachtung in einer Arbeit zu leisten. Gleichzeitig verhindert diese wenig fokussierte Anlage, dass die Erkenntnisse über eine oberflächliche Betrachtung hinausgehen. So bietet die Arbeit zwar einen Einblick in die beiden Themenfelder, schöpft das Potenzial der Fragestellung aber nicht aus. Nach der Lektüre stellen sich deshalb eine Vielzahl detaillierter Anschlussfragen. Nichtsdestotrotz kann man auf Basis dieser ersten Ergebnisse erkennen, dass gesundheitsbezogene InfluencerInnenkommunikation einen wichtigen Stellenwert im Portfolio 
der Gesundheitsinformationen einnimmt und ein umfassendes Verständnis dieser Kommunikation ein lohnendes Ziel für zukünftige Forschungsarbeiten darstellt.

Funding Open Access funding enabled and organized by Projekt DEAL.

Open Access Dieser Artikel wird unter der Creative Commons Namensnennung 4.0 International Lizenz veröffentlicht, welche die Nutzung, Vervielfältigung, Bearbeitung, Verbreitung und Wiedergabe in jeglichem Medium und Format erlaubt, sofern Sie den/die ursprünglichen Autor(en) und die Quelle ordnungsgemäß nennen, einen Link zur Creative Commons Lizenz beifügen und angeben, ob Änderungen vorgenommen wurden.

Die in diesem Artikel enthaltenen Bilder und sonstiges Drittmaterial unterliegen ebenfalls der genannten Creative Commons Lizenz, sofern sich aus der Abbildungslegende nichts anderes ergibt. Sofern das betreffende Material nicht unter der genannten Creative Commons Lizenz steht und die betreffende Handlung nicht nach gesetzlichen Vorschriften erlaubt ist, ist für die oben aufgeführten Weiterverwendungen des Materials die Einwilligung des jeweiligen Rechteinhabers einzuholen.

Weitere Details zur Lizenz entnehmen Sie bitte der Lizenzinformation auf http://creativecommons.org/ licenses/by/4.0/deed.de.

Dr. Anja Kalch ist wissenschaftliche Mitarbeiterin am Lehrstuhl für Kommunikationswissenschaft mit Schwerpunkt Rezeption und Wirkung, Institut für Medien, Wissen und Kommunikation an der Universität Augsburg. 